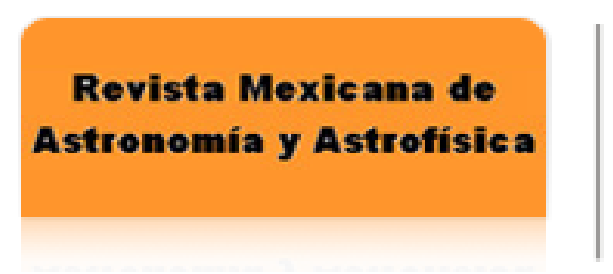

Revista Mexicana de Astronomía y Astrofísica ISSN: 0185-1101

rmaa@astroscu.unam.mx

Instituto de Astronomía

México

Cuevas, S.; Eikenberry, S. S.

FRIDA INTEGRAL FIELD UNIT OPTO-MECHANICAL DESIGN

Revista Mexicana de Astronomía y Astrofísica, vol. 42, 2013, p. 121

Instituto de Astronomía

Distrito Federal, México

Available in: http://www.redalyc.org/articulo.oa? $\mathrm{id}=57127862067$

How to cite

Complete issue

- More information about this article

Journal's homepage in redalyc.org

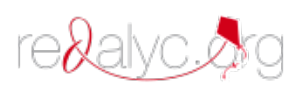

Scientific Information System Network of Scientific Journals from Latin America, the Caribbean, Spain and Portugal Non-profit academic project, developed under the open access initiative 
RevMexAA (Serie de Conferencias), 42, 121-121 (2013)

\title{
FRIDA INTEGRAL FIELD UNIT OPTO-MECHANICAL DESIGN
}

\author{
S. Cuevas ${ }^{1}$ and S. S. Eikenberry ${ }^{2}$
}

We present in this work the opto-mechanical design of each FRIDA IFU component, as well as the overall IFU structure.

The FRIDA IFU uses the "bolt-and-go" approach for diamond-turned image slicing FISICA IFU (Eikenberry et al. 2006). From a mechanical perspective, the FRIDA IFU mirrors and structures are similar or simpler than those of FISICA IFU.

FRIDA optical design is described by Cuevas et al. (2008). FISICA IFU mirrors blocks were manufactured on aluminum 6061-T6. New materials were studied for the FRIDA IFU to reduce the scattered light produced by the mirrors roughness (DeWitt et al. 2008).

We have developed a detailed opto-mechanical model of the FRIDA IFU mirror, including a realistic model of the diamond-turning tool and its cutting path for each slice. We presented the resulting FRIDA slicer mirrors in this work.

Each FRIDA component was designed on SolidWorks (SW) CAD software. In order to verify the mechanical design, each mirror or mirror block SW model was exported to ZEMAX optical design and ray-tracing was performed including testing for scattered light on the non optical surfaces. The complete ZEMAX FRIDA model was re-imported to SW and the ray tracing was included on the $3 \mathrm{D}$ modeling. Figure 1 shows a ray tracing on the neighborhood of the Field Mirrors block, the mirror array that form the FRIDA spectrograph pseudo slit. From the resulting 3D model the opto-mechanical mounts of the IFU were designed. Figure 2 shows a complete IFU isometric view. We can conclude FRIDA IFU is fully modeled, verified and ready for fabrication.

We are grateful to Grupo Santander (Spain) through Encuentros Astrofísicos Blas Cabrera (UNAM-IAC) and our home institutions for their support.

\section{REFERENCES}

Eikenberry, S. S., et al. 2006, Proc. SPIE, 6269, 62694L7 Cuevas, S., et al. 2008, Proc. SPIE, 7014E, 70146D DeWitt, C., et al. 2008, Proc. SPIE, 7014E, 70142X

\footnotetext{
${ }^{1}$ Instituto de Astronomía, Universidad Nacional Autónoma de México, Apdo. Postal 70-264, México, 04510 D.F., Mexico (chavoc@astroscu.unam.mx).

${ }^{2}$ Department of Astronomy, University of Florida, Bryant Space Science Ctr., Gainesville, FL 32611-2055, USA.
}

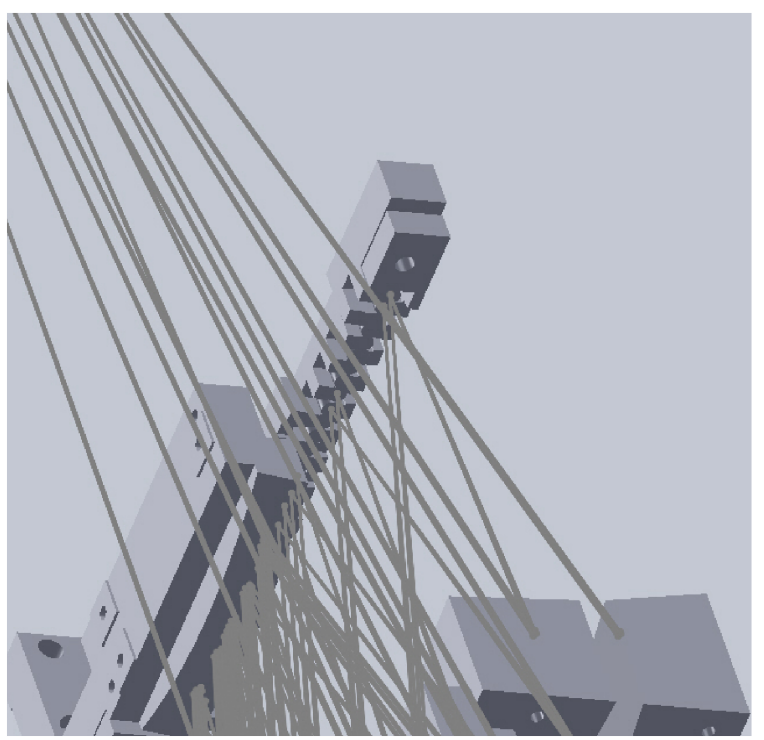

Fig. 1. Ray tracing around the neighborhood of the Field Mirror block. This block is the actual Solid Woks model.

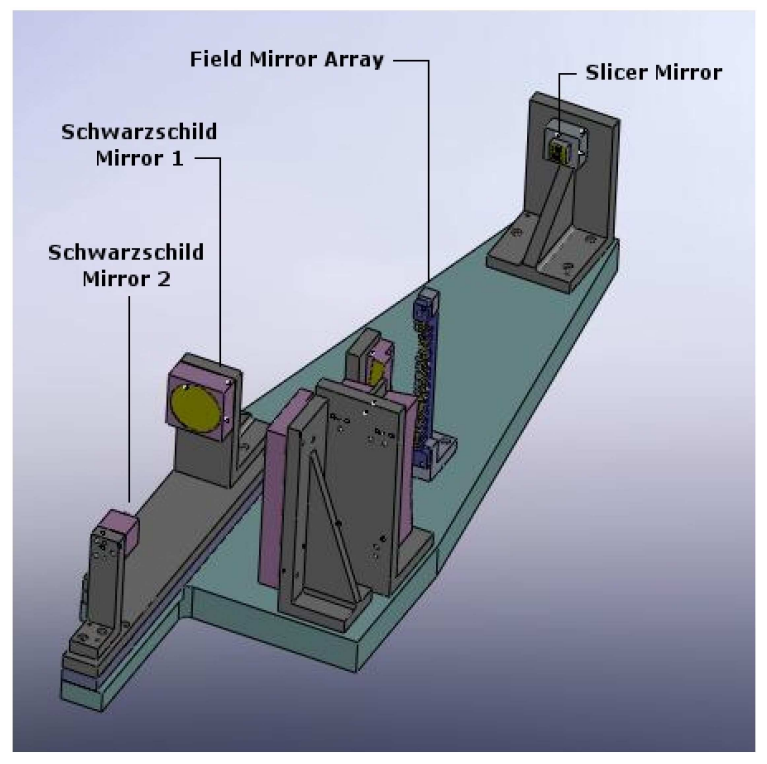

Fig. 2. 3D model of the FRIDA IFU. 